POLICY FORUM

CLIMATE POLICY

\title{
Identifying the policy space for climate loss and damage
}

\author{
Climate risk analysis must play a fundamental role
}

\section{By Reinhard Mechler ${ }^{1,2}$ and Thomas Schinko ${ }^{1,3}$}

C urrently planned greenhouse gas mitigation efforts would not prevent climate warming from going beyond $2^{\circ} \mathrm{C}$ as aspired to in the 2015 Paris Agreement (1), adding to climate-related impacts already under way (2). Although climate adaptation has been strengthened in the Paris Agreement, climate-related risks may exceed adaptation possibilities of communities and countries. To this effect, an important decision in the Paris Agreement was the endorsement of the Warsaw International Mechanism (WIM) for Loss and Damage (L\&D) (3). This established L\&D as a distinct pillar of climate negotiations, yet with an unclearly defined remit. With a policy framework yet to emerge, the 22nd Conference of the Parties (COP 22) of the United Nations Framework Convention on Climate Change (UNFCC) in November in Marrakesh will review the structure, mandate, and effectiveness of the WIM, first institutionalized in 2013. Risk science can provide a rationale and delineate a policy space for L\&D, composed of curative measures for unavoided and unavoidable impacts, and transformative measures for avoiding and managing intolerable risks.

Climate risks considered by the WIM are associated with extreme events-flooding, droughts, heat waves, and cyclones-and slow-onset impacts, including sea level rise and melting glaciers. Lacking official definition, losses have been associated with irreversibility-e.g., fatalities from climate events or households stuck in disaster-induced poverty traps, whereas damages refer to impacts that can be alleviated. A useful distinction has been made between avoidable, unavoided, and unavoidable L\&D (4).

IIIASA, Schlossplatz 1, 2361 Laxenburg, Austria. ²Vienna University of Economics and Business, 1020 Vienna, Austria. ${ }^{3}$ Wegener Center for Climate and Global Change, University of Graz, 8010 Graz, Austria.Email: mechler@iiasa.ac.at
Discussion of the WIM has been contested (5) and wide-ranging (6). The Alliance of Small Island States (AOSIS), whose members face substantial climate-related stress, initiated the debate more than two decades ago, proposing that parties with historically high emissions take responsibility via some sort of compensation instrument. With endorsement from other vulnerable developing countries, AOSIS has been arguing for an international mechanism basically made up of two components: support for risk management, including insurance efforts, and a rehabilitation or compensatory component addressing increasingly negative impacts (7). Many developed countries concur in principle on the need to help those suffering from climate change impacts but have been unwilling to accept notions of liability and have stressed the need to institutionalize incentives for tackling risks (8), as, for example, suggested by the 2015 Sendai Framework for Disaster Risk Reduction (DRR) (9). These parties suggested to cover the matter under adaptation, but the decision to have a standalone article of the Paris agreement (Article 8) refer to the WIM came about only after developing countries insisted that L\&D is distinct. Vulnerable countries celebrated the inclusion of the WIM in the agreement, while developed countries managed to include assurance that the WIM does not provide a basis for liability and compensation, which remain important background issues informing any potential implications of the WIM.

\section{A CLIMATE RISK SCIENCE PERSPECTIVE}

Assessments of climate change impacts have shifted in focus from academic to more operational, including engagement of multiple stakeholders via novel risk analytical methods $(10,11)$. Work had originally focused on tracing incremental impacts induced by global warming in order to identify "dangerous" anthropogenic interference for climate mitigation and adaptation purposes (IPCC's five Reasons for Concern) (12). An emergent perspective assesses risk as shaped by both

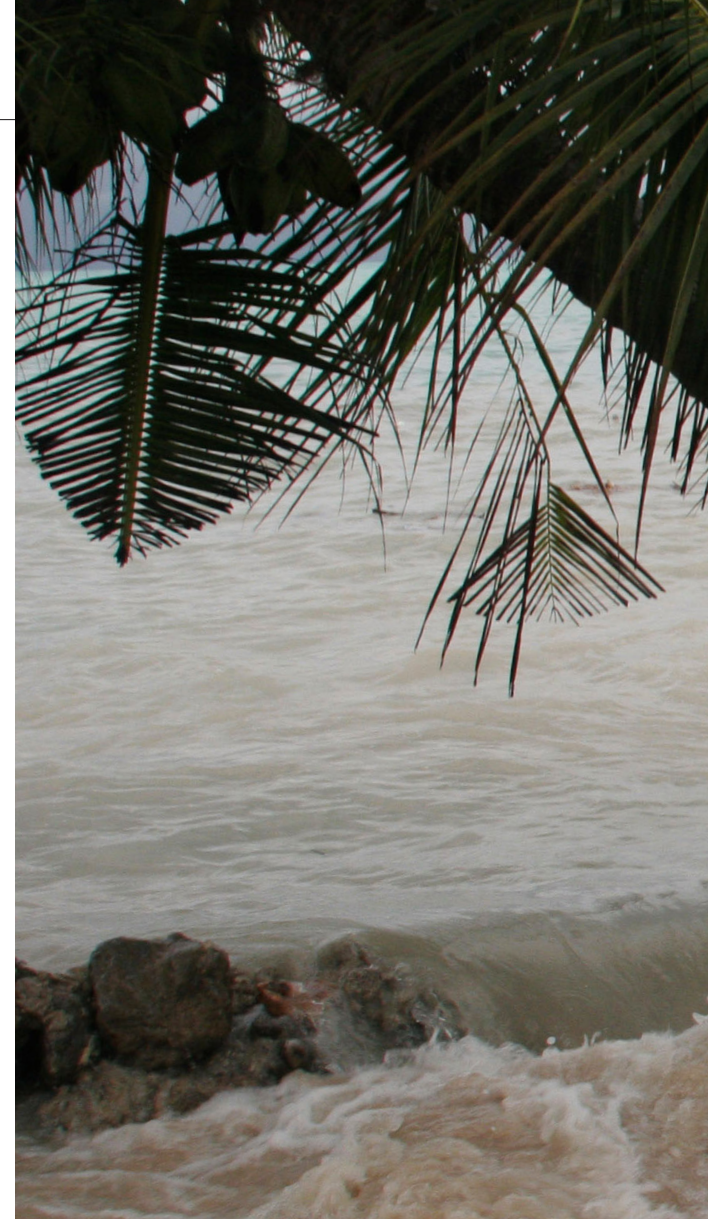

climate variability and climate change and seeks to support climate risk management (CRM) at different scales (13). CRM aligns DRR, focused on sudden-onset hydrometeorological and geophysical events, with climate change adaptation (CCA), tackling slow-onset and sudden-onset climate-related impacts. CRM's overall remit is to anticipate, avoid, prevent, and finance risks as well as absorb remaining impacts.

CRM includes concern for CCA/DRR gaps-the difference between what we want or need and what we actually have and implement in terms of funds, technology, and knowledge for risk management (14). Socially desirable levels of CRM will generally be less stringent than technically and physically feasible because of a number of negotiated trade-offs; e.g., costly flood protection, even in well-protected countries, ends at 50- to 100-year-flood return levels. Considerations of risk preference (15) have entered the debate (and IPCC) to provide classifications of risk as acceptable (no additional action necessary), tolerable (action required considering costs and other constraints), and intolerable (action required irrespective of constraints) (16).

Risk and risk tolerance are socially constructed. Although the IPCC, with medium and high levels of confidence, has identified many regions as facing substantial stress from climate change-exacerbated risks, what constitutes acceptable, tolerable, and intol- 


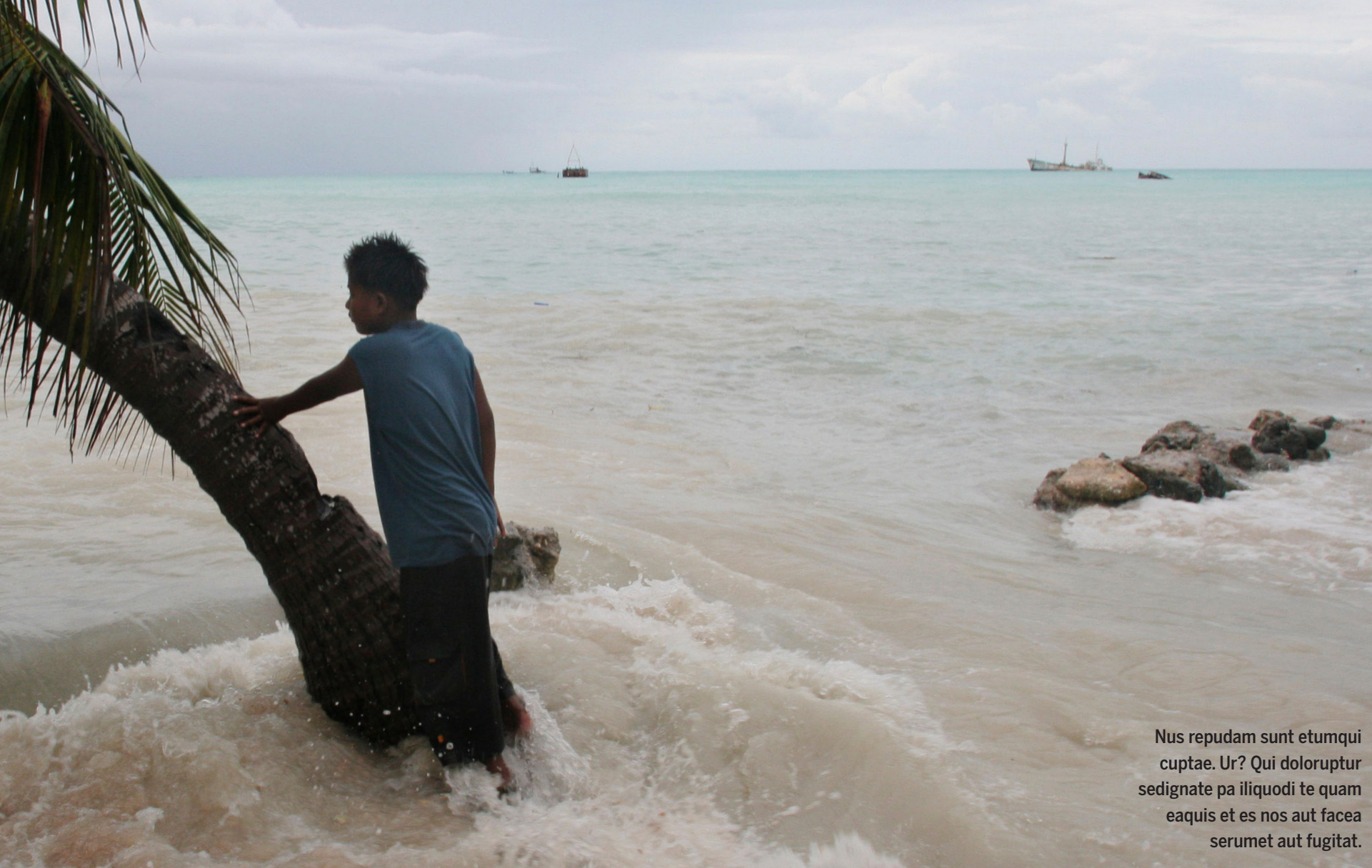

erable is strongly determined by social, cultural, and economic determinants and often requires joint subjective and expert deliberation before being submitted to allocating responsibilities. Risk analysis has developed analytical procedures for negotiating and segregating risk according to risk preference ("risk layering") that have been used in the insurance industry and are being applied to climate risk issues to help allocate risks to multiple actors at various scales (17).

An important dimension requiring more attention is climate justice, which scientifically is linked with attributing impacts to anthropogenic climate change, identifying harm-doing and burden-sharing of benefits and costs. Burden-sharing has been necessary because many vulnerable countries are in need of international support for tackling adaptation gaps. The international community has shared such obligations under principles of distributive justice (18), not requiring climate attribution of risks for generating international support, such as via the Global Facility for Disaster Risk Reduction. There is a need to consider compensatory justice due to the unequal distribution of historical and current emissions, as well as potential irreversible loss (19). The IPCC has attributed trends in slow-onset climate change and some climate extremes to anthropogenic greenhouse gas emissionsalso pointing out that risk causation is often multifactorial, with climate change and so- cioeconomic change as key risk drivers (10). Yet, although climate science has been making progress in climate attribution, linking anthropogenic emissions to impacts and to risks to people, property, and ecosystems remains complex, particularly for sudden-onset events (20). Both justice principles can be combined, yet balance needs attention. Many developing countries are worried that emphasis could be put on national responsibility for managing risks, rather than international law considerations associated with the "polluter pays" principle proportional to emissions contributions (21).

\section{THE L\&D RISK AND POLICY SPACE}

Aligning emerging analytical insight, we build and extend a schematic scenario assessment of risks and CRM conducted by IPCC's 5th assessment report at regional scale and for groups of countries for slow and suddenonset events (see the figure). The exemplary visualization applies the CRM framework to Small Island States faced with sea level rise and high-water level events. The IPCC considers today's risk as grave (medium), with further risk avoidance and reduction potential due to a considerable DRR/CCA gap (22). For example, technically and physically feasible DRR and CCA, such as elevating seawalls or maintaining coastal landforms, could bring risk down to acceptable levels today. Relying on national resources has generally not sufficed to reduce risk sufficiently in these countries, particularly for those in the Pacific, Caribbean, and Indian Ocean, and support from the international community has been required. In the near term, sea level rise will increase risks shaped by climate change and socioeconomic factors (increase of people and assets in harm's way). Although there is scope for DRR/CCA, some further risk is locked-in already, with serious cost implications (e.g., costs associated with upgrading coastal protection). The risk has increased, and part of this cannot be reduced anymore (not all land is being protected from flooding). We term these additional measures and necessary costs curative. Over time, risk is projected to increase. Challenges and costs will increase to well-tested CCA and DRR measures, and for strong warming $\left(4^{\circ} \mathrm{C}\right)$, risks become intolerable (23). Novel, transformative measures are needed, such as offering alternative livelihoods (e.g., switching from farming to services) and assisting with voluntary migration, as compared to curative support for forced migration.

Overall, with climate change amplifying risk, there is a legitimate case for international financial and operational support on L\&D to tackle avoidable but intolerable loss and damage by picking up part of the burden from DRR and CCA domains (the transformative part); with feasible risk reduction becoming limited over time, constraining societally desirable implementation pathways, measures for dealing with unavoided 


\section{Climate risk management options in small island states}

The risk and policy space for Loss and Damage as applied to risks from sea level rise in Small Island States Adapted from $(2,22,23)$. The scenarios identify classes of curative measures for unavoided and unavoidable impacts and transformative measures for avoiding and managing intolerable risks.

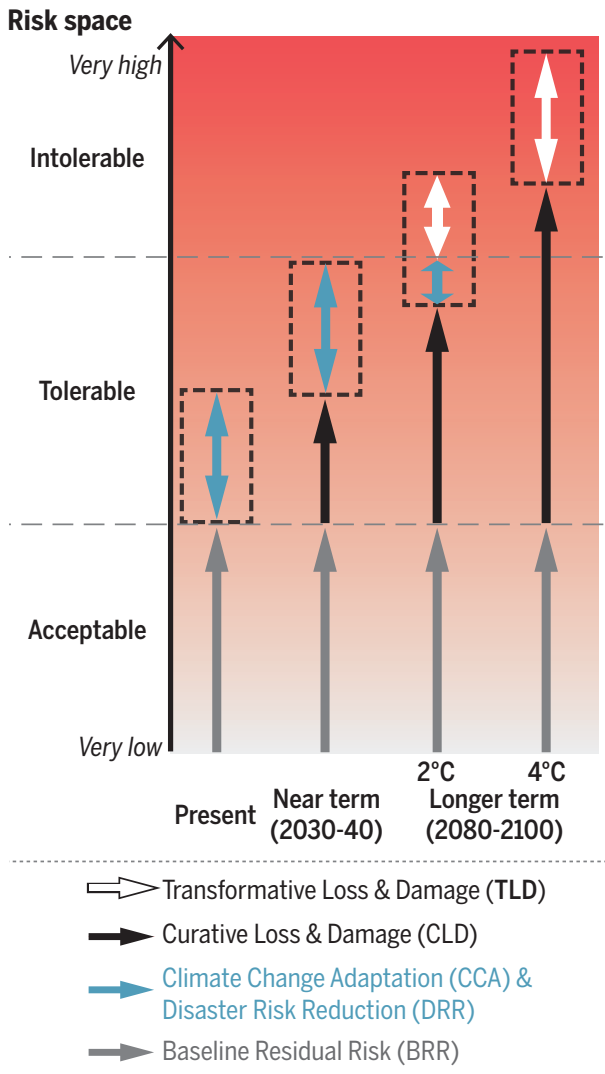

and unavoidable L\&D, both tolerable and intolerable, will need further attention (the curative part).

\section{BEYOND CURRENT DELIBERATIONS}

A broad climate risk analysis perspective may provide a framework for negotiating responsibilities and leading to principled action. Action already under way can be integrated into the framework. What we call transformative action is seeing attention with pledges made by the G7 to support the "climate risk insurance" initiative, which aims to provide insurance cover for climaterelated risks to an additional 400 million uninsured people in developing countries (24). This is connected to DRR support already granted for managing climate (and geophysical) variability via regional sovereign disaster risk insurance pools for Caribbean and Pacific island states, as well as countries in Central America and Africa. The transformative component to be further strengthened arises from efforts to broaden to comprehensive DRR, including risk prevention (e.g., coastal protection for low-lying islands) and preparedness (e.g., enhanced provision

\section{Options space}

TLD: Voluntary resettlement, alternative livelihoods

CLD: Involuntary migration and displacement

CCA: Maintenance and restoration of coastal landforms and marine and terrestrial ecosystems, improved management of soils and freshwater resources, appropiate building codes and settlement patterns

DRR: Seawalls, early warning, insurance

CLD: Absorbing increasing costs of DRR \& CCA with rising risk

BRR: no further measures

beyond those already implemented
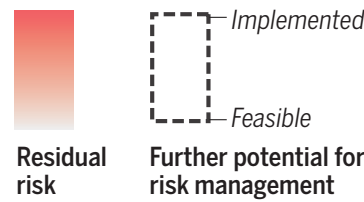

risk

Further potential for risk management

of functional flood early warning systems), and to build resilience, such as enabling farmers to take up-side risks (e.g., novel crop varieties with higher profits) in addition to better managing down-side risks.

It has been unclear whether the $\$ 300$ million pledged for climate insurance is truly additional. Thus better integrating CCA and DRR with transformative climate risk management efforts may ensure that intolerable risks are strongly acted upon via global solidarity, a keen demand by developing countries, rather than subsumed under country-driven approaches involving national responsibility. In adding L\&D efforts to CRM, the distinction from support for DRR and CCA might be that the WIM covers intolerable risk building on some sort of an attribution signal (an extreme climate facility, to be triggered by attributed changes in underlying climate variability, is being discussed for the Africa drought risk insurance pool) (25). What we call curative action has not really seen direct action, although there is nascent debate on a climate displacement coordination facility, which may deal with planned migration and legal status for involuntary displacement of communities that permanently have lost homes or homelands.

There is a very important role for science in this contested political debate. The WIM Executive Committee recently decided to set up task forces with suggested participation of scientists on risk management (including transformational approaches) and displacement. Major scientific challenges remain, in particular to help better understand the physical and social limits of adaptation. Translating the largely schematic climate risk assessment into quantitativequalitative projections of rising climate risks and associated costs of transformative and curative measures can help take the discourse on L\&D forward as well as motivate action toward the ambitious mitigation goals set in Paris.

\section{REFERENCES AND NOTES}

1. UNFCCC, Decision CP/2015/L.9 (UNFCCC, 2015)

2. IPCC, Climate Change 2014. Synthesis Report. Summary for Policymakers (Cambridge University Press, Cambridge, 2014).

3. UNFCCC, Decision 2/CP.19 (UNFCCC, 2013).

4. R. Verheyen, Tackling Loss \& Damage-ANew Role for the Climate Regime? (Climate and Development Knowledge Network, 2012)

5. K. van der Geest, K. Warner. Int. J. Global Warming 5,337 (2013).

6. R. James et al., Nat. Clim. Change 4, 938 (2014)

7. AOSIS, Proposal to the AWG-LCAMulti-Window Mechanism to Address Loss and Damage from Climate Change Impacts (AOSIS, 2008).

8. R. Roberts, S. Huq, Int. J. Global Warming 8,2141 (2015).

9. UN, Sendai Framework for Disaster Risk Reduction 2015-2030 (A/CONF.224/CRP.1,UN, 2015)

10. C. B. Fields et al. Eds., Climate Change 2014: Impacts, daptation, and Vulnerability, Part A: Global and Sectoral spects ( Cambridge Univ. Press, Cambridge, 2014). bnesetal., in (10), pp. 195-228.

12. J. Smith et al., in Climate Change 2001: Impacts, Adaptation, and Vulnerability, J. J. McCarthy et al., Eds. (Cambridge Univ. Press, Cambridge, , 2001), pp. 914-967.

13. W. Travis, B. Bates. Clim. Risk Manage. 1,1(2014).

14. M. Chambwera etal., in (10), pp.945-977.

15. German Advisory Council on Global Change (WBGU). World in Transition: Strategies for Managing Global Environmental Risks (Springer, Berlin, 1998).

16. K. Dow etal., Nat. Clim. Change 3,305 (2013)

17. R. Mechler et al.,Nat. Clim. Change 4, 235 (2014).

18. E.A. Posner, D. Weisbach, Climate Change Justice Princeton Univ. Press, Princeton, NJ, 2010).

9.). Roser et al., Clim. Change 133, 349 (2015).

2 0. C. Huggel etal., Nat. Clim. Change 6,901 (2016).

21. M. J. Mace, M. Schaeffer, Loss and Damage under the UNFCCC (Climate Analytics, Berlin, 2013).

22. L.A. Nurse etal. in (10) pp.1613-1654

23. UNFCCC, Decision FCCC/SB/2015/INF.1(UNFCCC, 2015).

24. GIZ, "Climate risk insurance for strengthening climate resilience of poor people in vulnerable countries" (GIZ, Eschborn, 2015)

25. J.Syroka, R.Wilcox,Planet@Risk 3,146 (2015).

\section{ACKNOWLEDGMENTS}

The authors acknowledge research funding from the project ENHANCE (Enhancing Risk Management Partnerships for Catastrophic Natural Disasters in Europe) funded by the European Commission under the Seventh Framework Programme (grant agreement no. 308438), as well as from the Zurich Flood Resilience Alliance. 